\title{
IMPERFECT KNOWLEDGE BASE SELF-ORGANIZATION IN ROBOTIC INTELLIGENT COGNITIVE CONTROL: QUANTUM SUPREMACY ON BIG DATA ANALYSIS
}

\author{
S.V. Ulyanov ${ }^{1,2}$, A.V. Shevchenko ${ }^{2}$, A.A. Shevchenko ${ }^{2}$, \\ A.G. Reshetnikov ${ }^{1,2,3, a}$ \\ ${ }^{1}$ Joint Institute for Nuclear Research - Laboratory of Information Technology, Dubna, 6 Joliot-Curie \\ St, 141980, Russia \\ ${ }^{2}$ Dubna State University - Institute of System Analysis and Control, Dubna, 19 Universitetskaya St, \\ 141982, Russia \\ ${ }^{3}$ Plekhanov Russian University of Economics, Laboratory of cloud technologies and Big Data \\ analytics; Moscow, Stremyanny Lane, 36, 117997, Russia \\ E-mail:agreshetnikov@mail.ru
}

The smart control design with secured achievement of information-thermodynamic trade-off interrelations is main goal for quantum self-organization algorithm of imperfect KB. Quantum genetic algorithm applied on line for the quantum correlation's type searching between unknown solutions in quantum superposition of imperfect knowledge bases of intelligent controllers designed on soft computing. Disturbance conditions of analytical information-thermodynamic trade-off interrelations between main control quality measures (as new design laws) discussed. Sophisticated synergetic quantum information effect in autonomous robot in unpredicted control situations) and swarm robots with imperfect KB exchanging between "master - slaves" introduced.A new robust smart controller on line designed from responses on unpredicted control situations of any imperfect KB applying quantum hidden information extracted from quantum correlation discussed. Within the toolkit of classical intelligent control, the achievement of the similar synergetic information effect is impossible.

Keywords: quantum genetic algorithm, intelligent cognitive robotics, quantum inference

Sergey Ulyanov, Andrey Shevchenko, AllaShevchenko, Andrey Reshetnikov

Copyright (C) 2021 for this paper by its authors. Use permitted under Creative Commons License Attribution 4.0 International (CC BY 4.0). 


\section{Introduction}

According to the definition of modern control problems, the achievement of required robustness property for a complex ill-defined control object models is possible with applying the computational intelligence toolkit. The goal of this article is the description of the applied aspects of developed intelligent design technology of robust knowledge bases (KB) [1-7] using the information synergy effects of quantum knowledge self-organization [2] in unpredicted and risky control conditions [5].

Four statements from quantum information theory and quantum thermodynamics are applied in this developed approach: 1) minimum entropy production rate principle of the system "control object + intelligent controller" that quadrantes the achievement of control goal with minimum of work waste in control object and in intelligent controller; 2) minimum information entropy principle for design intelligent cognitive controller that required minimum of initial information for intelligent controller action; 3 ) the amount of the work wasted on the extraction of hidden quantum information is less than the amount of work done on the received extracted quantum hidden information; and 4) the solution problem search of maximum extractable value work identical to a search of the minimum wasted entropy done on this work extraction[8-9]. The article task is the description of the IT-design process a robust sophisticated $\mathrm{KB}$ of intelligent cognitive controller that produce control force that satisfied to these requirements.

\section{Imperfect KB quantum self-organization process}

The role of specific quantum hidden information effects for smart control design described in [2]. The amount of hidden quantum information [2-4, 6] extracted from control classical states considered as the additional information-thermodynamic control force source.

In systems inspired by nature, robustness is determined by the natural process of selforganization [1-2]. The process of quantum self-organization of KBs, in which the robustness property is achieved, is shown in Fig. 1. Natural evolution consists of the following stages: 1) creating a template; 2) self-assembling; 3) self-organization (see level 2 in Fig. 1).

As is known from the theory of quantum computing, each quantum algorithm contains such unitary quantum operators as interference, superposition, entanglement (quantum oracle) and measurement classical operator (irreversible and used for measurement of quantum computations). The quantum fuzzy inference (QFI) model is based on the corresponding quantum operators and accumulates the principles of self-organization. A quantum self-organization control algorithm, based on QFI, is shown in Fig. 1.

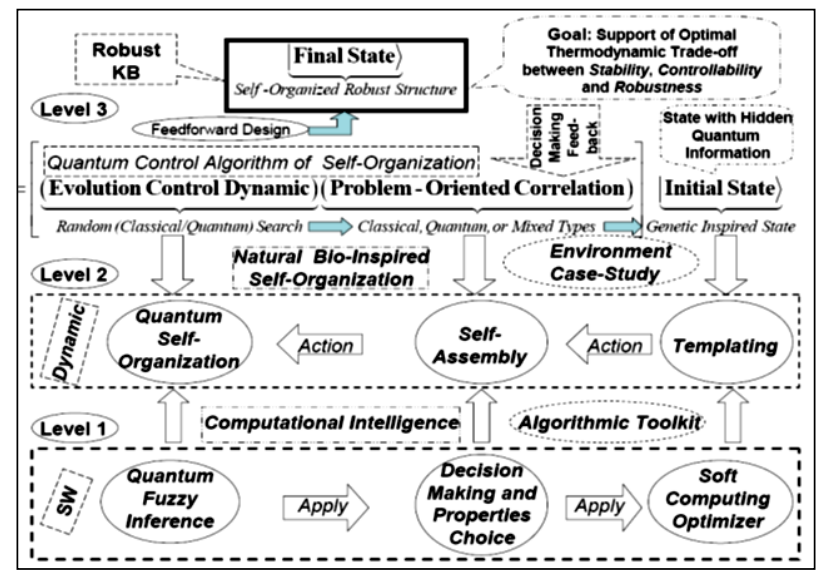

Figure 1. Quantum search hierarchical structure of self-organization robust KB's design system 
Thus, this quantum control algorithm uses the basic principles of quantum information theory, which are useful information resources of QFI. Based on these principles, it is possible to extract additional hidden information and eliminate the redundancy of information for the formation of a control action, thereby increasing the stability of the ICS, providing robustness and accuracy of control in conditions of uncertainty or poorly formalized description of the external environment. Consequently, the inclusion of stability in the architecture of the structure of an intelligent control system contributes to an increase in its efficiency.

\section{Benchmark's simulation of smart control with QFI}

In Figure 2 shows the results of an experiment of control in unexpected situations for an object "cart-double pole" and a 7 degrees of freedomredundant manipulator. The experiment compares the different controllers: PID controller, two fuzzy controllers (FC1, FC2) and three QFI controllers based on different types of correlations: Quantum-Time (Q-T), Quantum-Space (Q-S), Quantum-SpaceTime (Q-ST).

In the simulation and experiment, the structure of a robust ICS based on QFI (see Fig. 2) and QAG (see Figure 3) was used. Based on the training signal taken directly from the control object, using the QCOptKB ${ }^{\mathrm{TM}}$ software toolkit, a KB of $\mathrm{FC}$ was designed. An abnormal situation was simulated by a threefold delay in the feedback sensor signal.
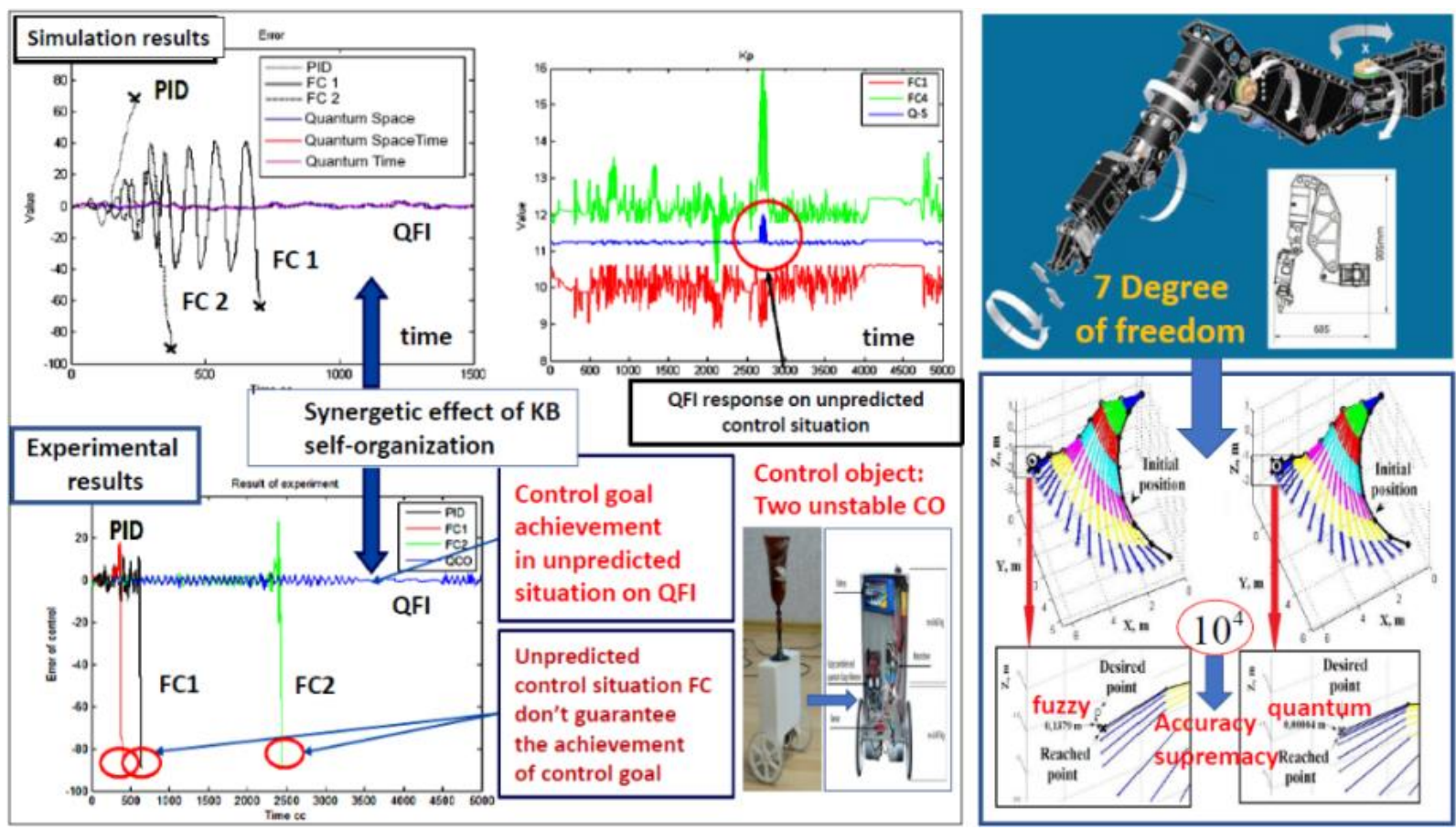

Figure 2. The experiment of control in unexpected situations for an object "cart-double pole" and a 7 degrees of freedomredundantmanipulator

The experimental results show that the accuracy of a quantum controller is more than 10,000 (see Fig. 2, right side) times higher than that of a controller based on soft computing. Under conditions of uncertainty, the controller based on soft computing dramatically increases the control error, thereby failing to achieve the control goal (see Table 1). Comparison of controllers shows the presence of a synergistic effect of self-organization in the design of robust KBs based on imperfect KBs of FCs. The control coefficients of the PID controller are based on the feedback of imperfect KB (see the "QFI block" in Fig. 1), forming a control action in online. This is achieved by extracting an additional information resource using QFI in the form of quantum information hidden in the classical states of the control action as a new control error of the output signal of an imperfect KB [1-2]. 
Table 11. Comparison of the different regulators

\begin{tabular}{ccccccc}
\hline \multirow{2}{*}{ Time, sec } & Cart motion, cm \\
\hline 1 & -1 & -1 & -1 & 1 & -1 & -1 \\
2 & 5 & 3 & 5 & 5 & 3 & 4 \\
3 & -35 & -4 & -26 & -4 & -2 & -3 \\
4 & 60 & 5 & 36 & 6 & 4 & 5 \\
5 & - & -5 & -60 & -5 & -4 & -7 \\
6 & - & 10 & - & 5 & 8 & 6 \\
7 & - & -14 & - & -4 & -6 & -9 \\
8 & - & 23 & - & 4 & 5 & 7 \\
9 & - & -32 & - & -6 & -8 & -3 \\
10 & - & 50 & - & 9 & 6 & 4 \\
11 & - & - & - & -9 & -4 & -7 \\
\hline
\end{tabular}

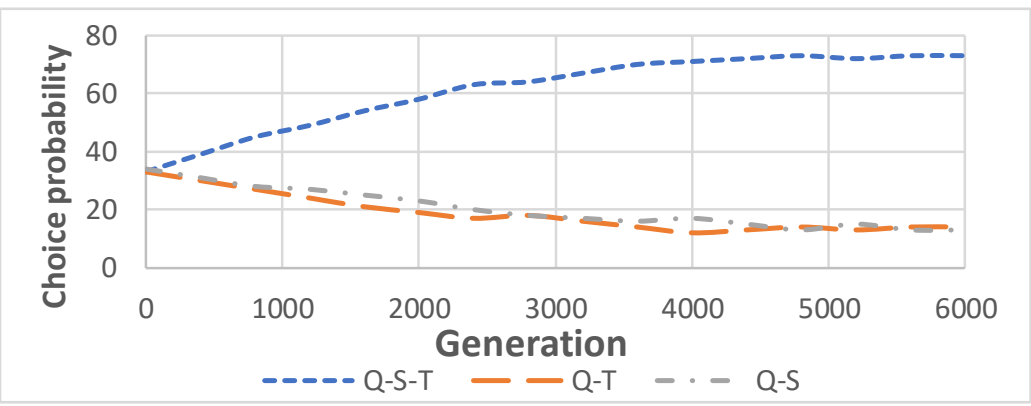

Figure 3. The result of the QGA

However, after 200 generations the probability of spatio-temporal correlations decreases to $60 \%$ (see Fig. 4).

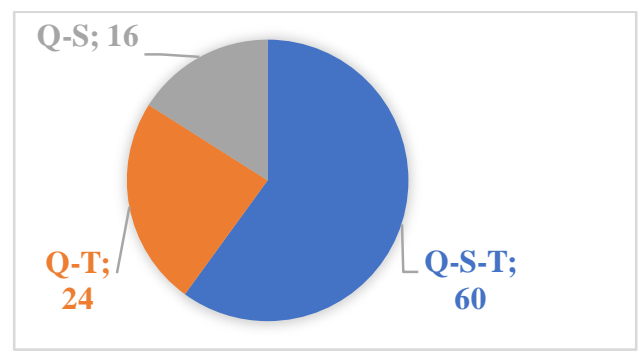

Figure 4 . The probability of spatio-temporal correlations after 200 generations

The described method is differed from others results described in [7-8].

\section{Conclusion}

This paper describes a method for designing intelligent control systems using a quantum algorithmic gate for quantum fuzzy inference based on a quantum genetic algorithm. This method in online allows to achieve global sustainability in the face of unforeseen management situations. Building on the computing power of classical computers, new types of quantum computational intelligence tools such as quantum and soft computing are used. The presented QFI model implements a new type of quantum search algorithm with the introduction of a quantum genetic algorithm, which makes it possible to design a robust ICS with classical nonlinear objects (such objects can be considered as a standard for testing the effectiveness of an ICS) control in conditions of global instability $[4,6-8,12]$ and can be used for big data analysis. Such a synergistic effect is achieved using hidden quantum information (as an additional resource), which obeys only the laws of quantum physics and has no analogues in classical physics. 


\section{References}

[1] Litvintseva L. V., Ulyanov I. S., Ulyanov S. V., Ulyanov S. S. Quantum Fuzzy Inference for Knowledge Base Design in Robust Intelligent Controllers. Journal of Computer and Systems Sciences International, 46(6), 908-961 (2007). doi: 10.1134/S1064230707060081.

[2] Litvintseva L. V., Ulyanov S. V. Intelligent Control Systems. I. Quantum Computing and SelfOrganization Algorithm. Journal of Computer and Systems Sciences International, 48(6), 946984 (2009). doi: 10.1134/S1064230709060112.

[3] Ulyanov S. V. Intelligent Robust Control System Based on Quantum KB-Self-organization: Quantum Soft Computing and Kansei / Affective Engineering Technologies. Springer International Publishing, 37-48, (2014). doi: 10.1007/978-3-319-11310-4_4

[4] Ulyanov S.V. Intelligent self-organized robust control design based on quantum/soft computing technologies and Kansei Engineering. Computer Science J. of Moldova, 21(2(62)), $242-279$ (2013).

[5] Ulyanov S.V. Self-organizing quantum robust control methods and systems for situations with uncertainty and risk. Patent US 8788450 B2, 2014.

[6] Ulyanov S. V. Self-organized robust intelligent control.Saarbrücken: LAP Lambert Academic Publishing. 412, (2015).

[7] Ulyanov S.V. Quantum relativistic informatics. LAP LAMBERT Academic Publishing, OmniScriptum GmbH \& Co. KG, (2015).

[8] Ulyanov S.V. Quantum Algorithm of Imperfect KB Self-organization Pt I: Smart Control Information-Thermodynamic Bounds. Artificial Intelligence Advances, 3(2): 18-43, (2021).

[9] Ahmadi B., Salimi S., Khorashad A.S., Kheirandish F. The quantum thermodynamic force responsible for quantum state transformation and the flow and backflow of information. SCIENTIFIC REPORTS, 9(8746), (2019) https://doi.org/10.1038/s41598-01945176-1.

[10] Ulyanov S.V., Litvintseva L.V. Design of self-organized intelligent control systems based on quantum fuzzy inference: Intelligent system of systems engineering approach. IEEE International Conference on Systems, Man and Cybernetics. 2005. Hawaii, USA, 10-12 Oct, 4: 3835-3840 (2005).

[11] Ulyanov S. V. Quantum fast algorithm computational intelligence PT I: SW / HW smart toolkit. Artificial Intelligence Advances, 1(1): 18-43, (2019).

[12] Korenkov V.V., Ulyanov S.V., Shevchenko A.A., Shevchenko A.V. Intelligent cognitive robotics. Pt. I: Quantum cognitive computing technologies. Kurs. (2021). 